Internist 2014 $\cdot 55: 1265-1266$

DOI 10.1007/s00108-014-3473-2

Online publiziert: 16. Oktober 2014

๑) Springer-Verlag Berlin Heidelberg 2014

\section{G. Hasenfuß}

Herzzentrum, Klinik für Kardiologie und Pneumologie, Universitätsmedizin Göttingen, Georg-August-Universität

\section{Internistische Intensivmedizin, Organunterstützung und Organersatz}

\section{Therapieoptionen bei Organversagen}

Die rasante Entwicklung der Medizintechnik in den vergangenen Dekaden hat insbesondere in der Intensivmedizin das Spektrum der Behandlungsmöglichkeiten erheblich erweitert. Im Zuge dessen hat sich auch die Prognose von Patienten mit lebensbedrohlichen Erkrankungen verbessert. Heute können lebenswichtige Organe mit schwersten Funktionsstörungen entscheidend unterstützt oder temporär ersetzt werden. Die Sinnhaftigkeit hängt allerdings entscheidend davon $\mathrm{ab}$, ob während des Organersatzes die zugrunde liegende Erkrankung behandelt werden kann. Diese Frage sollte der Entscheidung vorangestellt sein, ob eines der Therapieverfahren zum Einsatz kommt.

> Neue Verfahren der Organunterstützung oder des Organersatzes sollten möglichst in randomisierten Studien validiert werden.

Das Beispiel der intraaortalen Gegenpulsation (IABP) bei verschiedenen Formen des kardiogenen Schocks verdeutlicht dies. Über mehrere Jahrzehnte galt das Verfahren als "State of the Art" in der Behandlung des kardiogenen Schocks. Erst kürzlich konnte dann die randomisierte IABP-SHOCK-Studie bei über $600 \mathrm{~Pa}$ tienten mit kardiogenem Schock nach Myokardinfarkt zeigen, dass weder die 30-Tage-Mortalität noch die 12-MonatsMortalität durch dieses Verfahren verbessert werden kann $[1,2]$.

Wie T. Seidler allerdings in dieser Ausgabe von Der Internist zeigt, sind zwi- schenzeitlich zahlreiche mechanische Unterstützungsverfahren entwickelt worden, die für die Behandlung des kardiogenen Schocks und insbesondere von mechanischen Komplikationen nach Myokardinfarkt evaluiert werden. Das Spektrum reicht von der implantierbaren Mikroaxialpumpe bis zum vollständigen Linksherzersatz. Die Indikationen beinhalten die Überbrückung der Schocksituation bis zur weiteren Entscheidungsfindung („,bridge to decision“), die Überbrückung zur Transplantation (,bridge to transplantation") und die Dauertherapie („destination therapy“; [3]). Eine weitere Miniaturisierung wird in Zukunft ermöglichen, dass einige dieser Systeme katheterbasiert minimal-invasiv und schonend implantiert werden können.

Wie Wutznowski et al. berichten, entwickeln bis zu 70\% aller Patienten auf Intensivstationen ein Nierenversagen, das die Prognose ungünstig beeinflusst. Im Vordergrund steht die Prävention desselben. Ist diese allerdings nicht erfolgreich, kann in Abhängigkeit von der zugrunde liegenden Eliminationsstörung und der hämodynamischen Situation auf verschiedene Nierenersatzverfahren zurückgegriffen werden. Wutznowski et al. gehen auf Pathophysiologie, Diagnostik und Therapie des Nierenversagens ein und diskutieren die Differenzialindikation zur Nierenersatztherapie. Klassische Indikationen sind die konservativ nicht kontrollierbare Hypervolämie, die therapierefraktäre Hyperkaliämie, die metabolische Azidose, die Urämie sowie die Intoxikation mit dialysablen Substanzen [4]. Je nach ursächlicher Störung und hämodynamischer Stabilität wird ein intermittierendes Verfahren oder ein kontinuierliches Verfahren eingesetzt.

Wie Ott et al. zeigen, kommen auch beim Leberversagen Nierenersatzverfahren zur Elimination toxischer Metabolite zur Anwendung. Zellbasierte extrakorporale Systeme und die Hepatozytentransplantation befinden sich bereits in klinischer Erprobung.

Bei schwerstem Lungenversagen mit Hypoxämie und/oder Hyperkapnie stehen seit wenigen Jahren ebenfalls extrakorporale Unterstützungsverfahren zur Verfügung. Auf diese Thematik gehen Müller et al. näher ein. Je nach hämodynamischer Situation des Patienten werden pumpenfreie arteriovenöse und pumpengetriebene venovenöse Systeme eingesetzt. Erstere eignen sich zur Kohlendioxidelimination, während mit letzteren sowohl die Oxygenierung als auch die Kohlendioxideliminierung erfolgt. Erfahrene Zentren verzeichnen Überlebensraten von bis zu $60 \%$ auch bei schwerstem Lungenversagen [5].

Bergwelt-Baildon et al. stellen die gängigen Verfahren der Stammzelltransplantation zur Knochenmarkersatztherapie bei verschiedenen neoplastischen Erkrankungen, aber auch bei schweren aplastischen Anämien vor.

Es wird unschwer deutlich, dass die technische Entwicklung in den vergangenen Jahren die Möglichkeit der modernen internistischen Intensivmedizin dra- 
matisch verbessert hat. Bei aller Faszination muss sichergestellt sein, dass diese Verfahren nur in erfahrenen Zentren und nur nach kritischer Evaluation eingesetzt werden. Des Weiteren sollte ihre Wirksamkeit möglichst in randomisierten Studien überprüft werden.

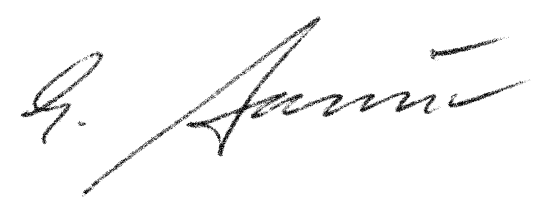

G. Hasenfuß

\section{Korrespondenzadresse}

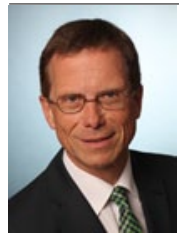

Prof. Dr. G. Hasenfuß

Herzzentrum, Klinik für

Kardiologie und Pneumologie,

Universitätsmedizin Göttingen,

Georg-August-Universität

Robert-Koch-Str. 40,

37075 Göttingen

hasenfus@med.uni-

goettingen.de

Interessenkonflikt. G. Hasenfuß gibt an, dass kein Interessenkonflikt besteht.

\section{Literatur}

1. Thiele H, Zeymer U, Neumann F-J et al (2012) Intraaortic balloon support for myocardial infarction with cardiogenic shock. N Engl J Med 367:12871296

2. Thiele H, Zeymer U, Neumann F-J et al (2013) Intraaortic balloon counterpulsation in acute myocardial infarction complicated by cardiogenic shock (IABP-SHOCK II): final 12 month results of a randomised, open-label trial. Lancet 382:1638-1645

3. McMurray JJV, Adamopoulos S, Anker SD et al (2012) ESC Guidelines for the diagnosis and treatment of acute and chronic heart failure 2012. Eur Heart J 33:1787-1847

4. Odutayo A, Adhikari NKJ, Barton J et al (2012) Epidemiology of acute kidney injury in Canadian critical care units: a prospective cohort study. Can J Anesth 59.934-942

5. Noah MA, Peek GJ, Finney SJ et al (2011) Referral to an extracorporeal membrane oxygenation center and mortality among patients with severe 2009 influenza A(H1N1). JAMA 306:1659-1668

\section{Förderpreis für Konzepte zur Verbesserung der intensivme- dizinischen Versorgungsquali- tät 2015}

Der vom Wissenschaftlichen Verein zur Förderung der klinisch angewendeten Forschung in der Intensivmedizin e.V. (WIVIM) ausgeschriebene Förderpreis in Höhe von $€ 3.000$,- wird für innovative Projekte und Projektvorhaben vergeben, die auf eine nachhaltige Verbesserung der intensivmedizinischen und intensivpflegerischen Versorgungsqualität abzielen. Hierbei werden vorzugsweise Projekte berücksichtigt, die anerkannte Instrumente des Qualitätsmanagements (PDCA-Zyklen) verwenden.

Der Förderpreis wird jährlich ausgeschrieben und vergeben. Um den Förderpreis können sich alle Teilnehmer des Symposiums Intensivmedizin + Intensivpflege bewerben. Berücksichtigt werden Projekt-Manuskripte in deutscher oder englischer Sprache, die bis zum 15. Dezember 2014 in elektronischer Form als PDF an kontakt@wivim.de eingereicht worden sind.

Für die Bewerbung sind ein formloses Anschreiben und eine kurze strukturierte Projektbeschreibung notwendig. Die Projektbeschreibung sollte neben der Thematik insbesondere das zugrunde liegende Konzept und die Zielstellung, die methodische Aufarbeitung, Eckpunkte der Durchführung und der anschließenden Evaluierung sowie Informationen zur Gesamtlaufzeit des Projektes enthalten.

Bei Fragen bitte an Prof. Dr. Gerlach, herwig.gerlach@vivantes.de wenden. Im Fall der Preisvergabe soll der Preisträger seine Ergebnisse, ggf. auch in Form eines vorläufigen Projektberichts, auf dem im darauffolgenden Jahr stattfindenden Symposium für Intensivmedizin + Intensivpflege präsentieren.

Die Preisvergabe nimmt die Jury während des Bremer Symposiums für Intensivmedizin + Intensivpflege 2015 vor.

Quelle: Wissenschaftlicher Verein zur Förderung der klinsch angewendeten Forschung in der Intensivmedizin e. V. Bremen (WIVIM), www.wivim.de

\section{Deutscher Schmerzpreis 2015 ausgeschrieben}

Die Deutsche Gesellschaft für Schmerzmedizin e.V. schreibt zusammen mit der Deutschen Schmerzliga e.V. jährlich den DEUTSCHEN SCHMERZPREIS - Deutscher Förderpreis für Schmerzforschung und Schmerztherapie - aus. Mit ihm werden Persönlichkeiten ausgezeichnet, die sich durch wissenschaftliche Arbeiten über Diagnostik und Therapie akuter und chronischer Schmerzzustände verdient gemacht oder die durch ihre Arbeit oder ihr öffentliches Wirken entscheidend zum Verständnis des Problemkreises Schmerz und den davon betroffenen Patienten beigetragen haben.

Verliehen wird der Deutsche Schmerzpreis im Rahmen des Deutschen Schmerz- und Palliativtages 2015 in Frankfurt/Main. Er wird von der Firma Mundipharma Vertriebsgesellschaft, Limburg, gestiftet und ist mit $€$ 10.000 ,-- dotiert. Nominierungen und Bewerbungen müssen bis spätestens 30 . November 2014 bei der Geschäftsstelle eingereicht werden. Die Wahl erfolgt durch eine unabhängige Jury und den wissenschaftlichen Beirat.

Quelle: www.dgschmerzmedizin.de 